Revista de la Escuela de Ciencias de la Educación, año 12, nRo. 11, vol. 2, julio a diciembre de 2016. Páginas 217235. ISSN 1851-6297 - ISSN 2362-3349 (En LíneA). LAS INICIATIVAS EN PRIMERA Infancia Bajo análisis. LaURA SANTILLÁN

\title{
LAS INICIATIVAS EN PRIMERA INFANCIA BAJO ANÁLISIS: ACTORES, RACIONALIDADES Y SENTIDOS EN TORNO A LA CRIANZA Y EL CUIDADO INFANTIL
}

\author{
Laura Santillán* \\ Universidad de Buenos Aires, Argentina. \\ laursantillan@gmail.com
}

Recibido: 15/03/2016 Aceptado: 10/05/2016

En este artículo el objetivo es abordar las premisas que se ponen en juego en torno a los niños y las niñas que transitan la primera edad de la vida entre cero a cinco años. Ahondaremos en el análisis de fuentes documentales y legislativas generadas por organismos estatales y no gubernamentales que participan del campo de intervención en Primera Infancia (1). Junto con el interés por los actores que intervienen, nos preguntamos por las racionalidades y saberes que construyen. Es nuestra hipótesis que, desde las voces que hegemonizan el campo de intervención, las premisas sobre la primera infancia fomentan un particular lenguaje del cuidado, la crianza y educación infantil. Este lenguaje se sustenta en el valor neutro de la ciencia, pero sin embargo devela sentidos morales y políticos tendientes a la modelación de hábitos en las familias. El análisis documental se inscribe dentro del trabajo de campo antropológico que venimos llevando adelante en el Gran Buenos Aires, Argentina.

Doctora en Antropología Social y Lic. en Ciencias Antropológicas por la Facultad de Filosofía y Letras, UBA. Investigadora dentro de la Carrera de Investigación en el CONICET (Categoría Adjunta) y en el Instituto de Ciencias Antropológicas de la Facultad de Filosofía y Letras, UBA. Docente Adjunta en el Departamento de Ciencias Antropológicas de la Facultad de Filosofía y Letras, UBA y profesora Titular en la Universidad Nacional de José C. Paz. Publicó el libro ¿Quiénes educan a los chicos? (Ed. Biblos, 2012), así como diversos artículos en revistas científicas nacionales y extranjeras sobre la problemática de la educación, la infancia y la desigualdad. 
Revista de la Escuela de Ciencias de la Educación, año 12, nRo. 11, vol. 2, Julio a diciembre de 2016. Páginas 217-

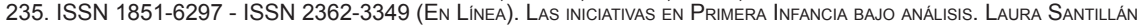

\author{
Palabras clave: \\ Primera Infancia - Cuidado Infantil - Regulación Moral - Postulados \\ Hegemónicos - Desigualdad.
}

\begin{abstract}
Analyzing the initiatives on Early Childhood: actors, rationalities and meanings regarding child care and child rearing

The aim of this article is to analyze the statements and meanings displayed around children who are at their early years of life. We will set out to conduct an analysis of documental and legislative sources produced by government and non -government agencies that take part in the field of interventions regarding Early Childhood, between or to five years. Along with our interest related to the intervening actors, we wonder about the rationalities and knowledge they construct. Our hypothesis is that considering the voices that are hegemonic in this field of intervention, the principles about early childhood encourage a certain language of caring, rearing and children's education. This language nourishes in the neutral value of science, but still, it shows moral and political meanings aimed at modeling family habits. The documental analysis is part of the anthropological fieldwork that we are carrying out in the suburbs of Buenos Aires, Argentina.
\end{abstract}

\title{
Keywords:
}

Early Childhood - Child Care - Moral Regulation - Hegemonic Statements Inequality

\section{Introducción}

En el presente trabajo nos centraremos en el análisis de las premisas y construcción de sentidos que se ponen en juego en iniciativas destinadas al cuidado y la educación de niños y niñas que transitan la primera edad de la vida, marcada por el momento previo al ingreso a la escolaridad básica. Desde los últimos años, somos de testigos de un importante desarrollo y expansión de acciones inscriptas dentro de lo que comienza a definirse bajo la categoría de atención a la "Primera Infancia". Aludimos a iniciativas que tienen lugar dentro y fuera del Estado e involucran de lleno a organismos internacionales y -en un amplio espectro- a organizaciones no gubernamentales.

Los enunciados relativos a la Primera infancia ${ }^{1}$ que hegemonizan hoy el campo de intervención -entre los que se destacan los provenientes de los organismos internacionales- develan una serie de cuestiones claves. Entre ellas, cuáles son las problemáticas que -en un contexto y momento determinado- son relevantes de atender, así también en cuanto a las características de las poblaciones que son destinatarias y sus necesidades. Según es posi- 
Revista de la Escuela de Ciencias de la Educación, año 12, nRo. 11, vol. 2, Julio a diciembre de 2016. Páginas 217235. ISSN 1851-6297 - ISSN 2362-3349 (EN LínEA). LAS INICIATIVAS EN PRIMERA INFANCIA BAJO ANÁLISIS. LAURA SANTILLÁN

ble advertir, en la contemporaneidad, un consenso sostenido con fuerza tiene que ver con definir a la atención en Primera Infancia como una intervención necesaria, cuando no epocalmente prioritaria.

Sin desmerecer la relevancia que tiene desarrollar estrategias destinadas a la atención de esta u otra franja etaria (2), en este escrito nos disponemos a analizar algunas premisas centrales que sustentan buena parte de los planteos programáticos en el tema. Aludimos a planteos plasmados, entre otros, en documentos y material escrito y que -aún con la diversidad de los agentes que intervienen- contienen ciertas líneas de unicidad entre sí.

Dentro de la literatura académica, podemos encontrar un conjunto amplio de trabajos cuya producción va al compás del desarrollo creciente de las iniciativas en Primera Infancia (Young \& Fujimoto-Gómez, 2003, Uamyahara, 2004, Jaramillo, 2009). En estos trabajos, algo a resaltar -aunque no en todos- es la marcada línea de continuidad que construyen respecto a los planteos más generales. Desde allí, es posible advertir el modo en que se asumen y dan por sentado algunos presupuestos. No es el caso de otros trabajos que, del mismo modo que intentaremos aquí, problematizan las premisas que se instalan como preponderantes (Rosemberg, 2002, Cardarelli, 2005, Goncalves Nunes, 2009).

La propuesta es avanzar hacia un análisis que contribuya a sistematizar -al menos como primer paso- los discursos que hegemonizan el campo de intervenciones en primera infancia. El interés es hacer inteligibles algunas de las aristas centrales que se producen en la contemporaneidad en torno al cuidado y la educación de los primeros años de la vida. Algunos interrogantes a los que aludiremos tiene que ver con conocer: ¿Quiénes son los actores que integran el campo de intervención en Primera Infancia? ¿Cuáles son sus condiciones de emergencia? ¿Cuáles son los discursos y saberes que se construyen? ¿Qué buscan ordenar -en clave de la vida y las prácticas sociales de sus destinatarios- estos discursos y saberes?

Es nuestra hipótesis que la conformación de este campo, aún en vista de las distintas voces que lo integran, devela la marcada preeminencia que tienen algunos actores -como los organismos internacionales-en su capacidad para fijar acentos y directrices en el tema. Siempre en referencia a los planteos tendientes a hegemonizar el campo, cabe decir que son directrices que sobresalen por su pretensión univerzalizante y que -desde un lenguaje neutral y objetivo- imponen valores y normas morales orientadas a la conducción de comportamientos. Influidos por los aportes de otros autores, entendemos a estas orientaciones en clave de regulación moral (Corrigan y Sayer, 2007), esto es como proyecto de normalización que opera volviendo natural y obvio aquello que en realidad es un conjunto de premisas ontológicas y epistemológicas configuradas con base a una determinada concepción de la sociedad y/o el orden social. Finalmente, en función a los modos en que hoy se cons- 
Revista de la Escuela de Ciencias de la Educación, año 12, nRo. 11, vol. 2, julio a diciembre de 2016. Páginas 217235. ISSN 1851-6297 - ISSN 2362-3349 (EN LINEA). LAS INICIATIVAS EN PRIMERA INFANCIA BAJO ANÁLISIS. LAURA SANTILLÁN

truyen los discursos y saberes sobre el tema, entendemos que uno de los efectos de las directrices sobre la primera infancia es realzar el lugar de las familias y comunidades de origen de los niños y niñas como causa y objeto de las intervenciones.

Para las reflexiones que siguen nos basaremos en el análisis del corpus documental que fuimos organizando en el marco de la indagación. Este corpus está formado por documentos legislativos y normativos provenientes de organismos estatales y no gubernamentales, así como por folletería y boletines producidos por los distintos actores puestos en juego. Para el trabajo con las fuentes nos sustentamos en aportes provenientes del análisis del discurso (Fairclough, 1993, Voloshinov, 2009). Desde estos planteos, partimos de una concepción amplia del discurso que lo comprende trascendiendo su función meramente referencial: el discurso es práctica social y se construye en las intersecciones que se producen entre lengua, cultura y poder (Fairclough, 1993). Asimismo, al trabajo con las fuentes secundarias, sumamos los registros provenientes de la observación participante que desarrollando en el transcurso del trabajo de campo que comenzamos en el año 2009 en barrios populares del Gran Buenos Aires, Argentina (3). En el análisis que sigue, el interés por develar los sentidos que se construyen en torno a la primera infancia, se inscribe y tiene como legado teórico a la tradición comprensivista e interpretativista desarrollada dentro de las ciencias sociales, y en particular en la Antropología social (Rockwell, 2009). Desde estos postulados, la realidad social es comprendida como un mundo continuamente construido y dotado de sentido (significación) que se forja en virtud a una continua disputa por la fijación de acentos (contenidos y connotaciones) por parte de diversos agentes y actores sociales con desigual peso de influencia (Marcus y Fischer, 2000).

\section{Polifonía: los actores en la atención a la primera infancia y la administración del riesgo}

El término polifonía refiere a la música en que intervienen varias voces e instrumentos, que ejecutan cantos distintos pero armónicos. Elegimos este término para titular este apartado ya que cuando nos disponemos al conocimiento de las iniciativas que van referenciando su trabajo en la Primera infancia, una de las cuestiones con la que nos encontramos es con la presencia de una multiplicidad de actores que portan su propia voz, a la vez que -tales voces heterogéneas- muchas veces conviven y quedan articuladas a través de procesos y sentidos que las aúnan.

A saber, en relación a la indagación etnográfica en los barrios populares que comprenden nuestra investigación, nos fuimos poniendo en contacto con una serie de propuestas y acciones que en el transcurso del tiempo fueron focalizando -in crescendo- su intervención en la atención a los primeros años 
Revista de la Escuela de Ciencias de la Educación, año 12, nRo. 11, vol. 2, Julio a diciembre de 2016. Páginas 217235. ISSN 1851-6297 - ISSN 2362-3349 (EN LINEA). LAS INICIATIVAS EN PRIMERA INFANCIA BAJO ANÁLISIS. LAURA SANTILLÁN

de la vida de niñas y niños. Jardines comunitarios, Centros de Desarrollo Infantil, merenderos, comedores, junto con otras propuestas estatales y autogestivas en los barrios (a cargo de voluntarios y militantes sociales ó de los propios pobladores) despliegan acciones destinadas a los niños y las niñas entre los 0 y 5 años, es decir que no concurren a la escolaridad básica de la primaria. El conocimiento y tránsito por estos espacios, nos puso de cerca de un importante número de interlocutores, entre los cuales se destacan maestras jardineras tituladas, educadoras populares no tituladas y también adultos que asumen el lugar de responsables de la atención: madres cuidadoras, vecinas, vecinos.

Con algunas diferencias, pero también importantes continuidades, una cuestión importante a destacar es la serie de preocupaciones que educadoras/es y cuidadoras/es nos fueron compartiendo a lo largo del tiempo. Sea cual fuere su campo de intervención, a lo largo de la indagación no nos ha pasado por alto las reiteradas alusiones acerca de lo crucial que significa su tarea, sobre todo en relación a la atención de los niños/as en sus primeros años como marcatorio para la vida futura, el desarrollo cognitivo y emocional:

\begin{abstract}
"Ahora tenemos una gran responsabilidad al recibir a los más chicos, nuestro trabajo impacta en el desarrollo y la vida futura" (docente del Nivel inicial en ejercicio).

"En los cursos aprendemos lo importante de atender los primeros años porque es en esos años que se determina todo, la estabilidad emocional, el desarrollo del cerebro, por eso hacemos un trabajo integral" (Responsable de merendero y centro infantil comunitario).
\end{abstract}

Algo que se pone de relieve -si no en todas, en buena parte de las intervenciones e iniciativas que conocimos- es la existencia de un lenguaje sobre el cuidado de los niños y las niñas que tímidamente $-\mathrm{y}$ en algunos casos con más contundencia- plantea algunas correspondencias con formas que se instalan acerca del tema. Esto no implica, vale mencionarlo, que quiénes llevan adelante las acciones realicen una recuperación y adhesión lineal a los planteos que provienen de esferas más generales. Hacia donde queremos detenernos es en el modo en que -en todo caso- una serie de postulados se tornan predominantes en la construcción y fijación de sentidos que comienzan a hegemonizar el campo de intervención. Aludimos a una profusa producción que se materializa en cartillas, folletos y documentación provenientes de diversas fuentes y usinas. Tal como veremos, en estas producciones es posible advertir la presencia estatal, aunque también -y muy fuertemente- la de las agencias que están por fuera del Estado.

A modo de ordenar el panorama de intervenciones, debemos decir que, 
Revista de la Escuela de Ciencias de la Educación, año 12, nRo. 11, vol. 2, julio a diciembre de 2016. Páginas 217235. ISSN 1851-6297 - ISSN 2362-3349 (EN LINEA). LAS INICIATIVAS EN PRIMERA INFANCIA BAJO ANÁLISIS. LAURA SANTILLÁN

en relación a las acciones del Estado, en el momento que realizamos nuestro trabajo de campo, las iniciativas destinadas a la primera infancia en nuestro país, se llevaban adelante a través de un conjunto amplio de Programas. Entre ellos se destaca el Programa Nacional Primeros Años, que se crea en el año 2005 en el ámbito del Consejo Nacional de Coordinación de Políticas Sociales, a través de un convenio firmado entre los Ministerios de Desarrollo Social, Salud y Educación de la Nación $n^{5}$. Asociadas a estas propuestas, al momento de realizar nuestra indagación, estaban vigentes el Programa nacional de Desarrollo Infantil y el Programa "Creciendo Juntos" (Ministerio de Desarrollo Social). Entre los objetivos de estos programas se encuentra el interés por fortalecer el crecimiento y desarrollo nutricional, emocional, social y motriz de niños y niñas, desde su nacimiento hasta los 4 años y es en función de este objetivo que se ha fomentado la realización de cursos de formación y capacitación destinados a educadores, madres cuidadoras, cocineros, nutricionistas y profesionales de diversas especialidades que acompañan el acceso de los niños a sus derechos.

Sin entrar, al menos por el momento, en el detalle de los sentidos y acentos que estos programas van poniendo en juego (en torno a la crianza, el desarrollo de los niños), es importante señalar que en nuestro país, el desarrollo de estas propuestas, no se da de ningún modo de manera aislada. Por el contrario, que se desenvuelve al unísono con iniciativas protagonizadas por el resto de los estados nacionales de la región y también en el mundo.

En nuestra región de Latinoamérica -e insistimos también por fuera de ella (6)-, desde por los últimos quince años a esta parte, los distintos países vienen desarrollado un conjunto de propuestas y programas destinados a la franja etaria mencionada como Primera infancia. En función de nombrar algunas experiencias, para el caso de Brasil, son ejemplos de ello las propuestas nacionales y federales que ha generado este país tales como el Programa Brasil Cariñoso y Bolsa Familiares, Programa Primera Infancia Mejor. En países como Colombia, a diferencia de Brasil, el objetivo en estos últimos años fue articular todas las propuestas bajo una sola política pública. Ejemplo de esta política nacional es el programa Colombia por la Primera Infancia. En Chile, desde el año 2006 se ha venido implementando el Programa Chile Crece Contigo, cuyo principal objetivo es acompañar y hacer un seguimiento personalizado de la trayectoria de desarrollo de los niños y las niñas hasta su ingreso al pre kinder (alrededor de los 4 o 5 años de edad). Este programa, cabe decir, recupera en buena medida las directrices formuladas en el programa Uruguay Crece Contigo, que se propone garantizar los procesos de cuidado y protección de mujeres embarazadas y el desarrollo integral de niños y niñas menores de 4 años. Continuando con este reconocimiento, en otros puntos del continente -la zona norte de Sudamérica y Centroamérica-, programas como el ecuatoriano Creciendo con Nuestros Hijos, el Programa 
Revista de la Escuela de Ciencias de la Educación, año 12, nRo. 11, vol. 2, julio a diciembre de 2016. Páginas 217235. ISSN 1851-6297 - ISSN 2362-3349 (EN LínEA). LAS INICIATIVAS EN PRIMERA INFANCIA BAJO ANÁLISIS. LAURA SANTILLÁN

Amor de Nicaragua y el Plan Quisqueya Empieza Contigo de la República Dominicana son también ejemplo de las intervenciones en Primera Infancia. La enumeración de estos programas nos permite advertir sobre la importancia que asume el recorte etario de los primero años de la vida a nivel geopolítico regional.

Ahora bien, ¿cómo explicar esta sucesión de medidas que -en la mayor parte de la región- se producen de manera simultánea? Sin descuidar los puntos de partida y los marcos políticos e ideológicos singulares que condujeron y conducen a cada país y región a orientar sus legislaciones y áreas programáticas hacia la atención a los primeros años de la vida (7), resulta imposible desvincular a estas decisiones de acuerdos trasnacionales (vinculantes y no vinculantes). Al respecto, la emergencia de un número importante de las programaciones de los distintos estados nacionales de nuestra región en Primera Infancia tiene como referencia una serie de reuniones y concertaciones internacionales. Entre ellas es ineludible la Conferencia Mundial conocida como "Educación para Todos", que se llevó adelante en Jomtien (Tailandia) en 1990, liderada por la UNESCO. Esta reunión, que contó con la participación de 92 estados, instó a la necesidad de alcanzar la concertación de todos los sectores y actores que integran cada uno de los contextos nacionales para el alcance universal de la educación básica. En relación con el tema que nos convoca, será recién en el Foro Mundial de Educación realizado en Dakar, Senegal, en el año 2000, cuando se acuerda que la Primera Infancia se incluya de manera explícita dentro del esquema de la "educación básica" (8). En relación con esta población, se insta a los estados nacionales a proporcionar un nuevo y más amplio margen político y social a los distintos actores que intervienen en la educación, en particular a la sociedad civil, apelando a la experiencia y el papel decisivo que ésta juega o debería jugar (UNESCO, 2000).

Por tanto, en referencia a la polifonía (de voces y actores) presentes en las intervenciones contemporáneas acerca de la primera infancia, debemos hacer ingresar al análisis -además de los Estados nacionales- a una profusa producción trasnacional. Dicho de otro modo, la acciones contemporáneas de atención a los primeros años de la vida, no pueden desprenderse de ciertas racionalidades y tecnologías globales de gobierno, y de las maneras que hoy se concretizan determinadas formas de gobierno y administración sobre las poblaciones (Rose, et al, 2006).

En relación a las referencias que traemos aquí, los aportes de Michel Foucault (2008) resultan muy relevantes. Los planteos teóricos que el autor forjó acerca de las formas contemporáneas de gobierno, nos permite comprender cómo en determinado momento de la historia moderna se instaura toda una economía moral y una necesidad de administración de las poblaciones A la vez, es en el marco de esa economía moral y gobierno que aspectos 
Revista de la Escuela de Ciencias de la Educación, año 12, nRo. 11, vol. 2, julio a diciembre de 2016. Páginas 217235. ISSN 1851-6297 - ISSN 2362-3349 (EN LINEA). LAS INICIATIVAS EN PRIMERA INFANCIA BAJO ANÁLISIS. LAURA SANTILLÁN

reconocidos como parte de la vida íntima (nacimientos, nivel de la salud, la duración de la vida, la muerte) quedan ligados a formas más generales del poder. En ese marco, sin más, es posible advertir cómo algunos hechos de la vida entran a la historia, ó lo que puede ser dicho en otras palabras: algunos fenómenos propios del desarrollo de las poblaciones comienzan a ser administrados y regulados a través de programas y tecnologías específicas (Foucault, 2008).

Siguiendo estos aportes, entendemos que las intervenciones y tematizaciones contemporáneas en relación a la Primera Infancia ponen de manifiesto algunas coordenadas básicas de las formas en que en la contemporaneidad se resuelven las intervenciones sobre determinadas poblaciones y grupos sociales. Al respecto, nos iluminan a introducirnos en preguntas tales: 1) ¿Quiénes deben ser hoy administrados? 2) ¿Por qué? 3) ¿Cómo?

Como es posible advertir desde las fuentes trabajadas, la difusión y expansión de un conjunto de políticas y programas destinados a un grupo hiper específico -como es el de la primera infancia- da cuenta sobradamente de la construcción acerca de cuál es la población sobre la cual hoy resulta imperioso intervenir. En el marco de la multiplicidad de voces que participan, las fundamentaciones van por carriles -al menos en principio- que portan singularidades propias.

En relación a los organismos de financiamiento multilaterales (Banco Mundial, BID), las argumentaciones en cuanto a la necesidad de intervenir en primera infancia se sustentan sin demasiados tapujos en el cálculo de las tasas de retorno a las inversiones en capital humano. Desde la perspectiva que sustentan estos organismos, emplear dinero en Primera infancia implica -sin más intermediaciones- el retorno positivo de lo invertido. En relación con esto, para el Banco Mundial, las respuestas a las preguntas sobre en quién invertir y por qué se destacan por no tener grises. Tal como señala el informe regional del organismo del año 2009:

Los DPI (Programas de desarrollo en la Primera Infancia) constituyen una manera eficaz en función de los costos de reducir la pobreza y la desigualdad en América Latina y el Caribe a mediano y largo plazo, en un momento en que la crisis financiera mundial continua complicando las economías de la región... [...] Los datos empíricos que aporta el nuevo informe demuestran que las intervenciones en la primera infancia son eficientes en términos económicos y tienen mayor rentabilidad que las inversiones que se hacen con posterioridad en jóvenes desfavorecidos [...]

Desde hace mucho que está comprobado que invertir en la gente es rentable. (Pamela Cox, Vicepresidenta del Banco Mundial para América Latina y el Caribe, Banco Mundial, 2009). 
Revista de la Escuela de Ciencias de la Educación, año 12, nRo. 11, vol. 2, julio a diciembre de 2016. Páginas 217235. ISSN 1851-6297 - ISSN 2362-3349 (EN LínEA). LAS INICIATIVAS EN PRIMERA INFANCIA BAJO ANÁLISIS. LAURA SANTILLÁN

Ahora bien, además de los organismos de financiamiento, desde agencias internacionales como UNESCO (9) y UNICEF (10) se formulan también propuestas de intervención y supervisión sobre el modo en que los estados y gobiernos nacionales deben encarar sus acciones sobre la Primera Infancia. En el caso de las agencias multilaterales no gubernamentales, el argumento sobre las mejorías en las tasas de retorno a la inversión no aparece con el mismo protagonismo que en los organismos de financiamiento. Progresivamente, desde los últimos años, estas agencias han venido estructurando sus argumentos sustentándose en un paradigma proteccionistas, afín con la declaración de la Convención Internacional de los Derechos del Niño.

Ahora bien, en relación a estos últimos organismos, hay que decir que, en el marco de referencia de los derechos, buena parte de sus argumentaciones se sostienen en una serie de postulados acerca del desarrollo de los niños en los cuales se insiste en una edad crucial para el crecimiento y la maduración cognitiva y emocional, como es la que va de los 0 a los 3 años. Tal como desarrollaremos en el próximo apartado, atender y problematizar estas cuestiones resulta muy relevante por los efectos sociales y políticos que contienen.

Con este trasfondo, volvemos nuevamente a las preguntas que nos habíamos formulado. Si avanzamos en el quiénes deben ser hoy administrados, continuamos ahondando en cuanto al por qué y al cómo. Tal como daremos cuenta en lo que sigue, algo que se va advirtiendo es el modo en que las argumentaciones sobre la atención de los primeros años de la vida se van sustentando en una idea de riesgo, que -como exponen otros autores (Rose, et al, 2006)- más que considerarlo como una cuestión intrínsecamente real, debemos considerarlo como una manera particular en la que los problemas son vistos, imaginados o enfrentados.

\section{Construcciones de sentidos: sobre aquello que buscan ordenar las propuestas que hegemonizan el campo de intervención}

Tomadas en conjunto, las propuestas contemporáneas destinadas a la primera infancia - aun en vista de sus distintas procedencias- presentan algunas coordenadas comunes. Hoy por hoy-dejando a un lado en este punto a los organismos multilaterales de financiamiento- prácticamente todos los anuncios y programas relativos a la Primera Infancia toman como marco de referencia a la Convención de los Derechos de los Niños. Es decir, la proclamación de los niños y las niñas como sujetos de derechos constituye el horizonte de buena parte de las políticas e intervenciones vigentes. El lenguaje de los derechos constituye un condensar de sentidos y un productor político-cultural potente en la legitimación de las muchas de las propuestas programáticas.

Unida con el enfoque de los derechos, otra continuidad entre las distintas iniciativas es la referencia a la necesidad de que la atención a la primera 
Revista de la Escuela de Ciencias de la Educación, año 12, nRo. 11, vol. 2, Julio a diciembre de 2016. Páginas 217235. ISSN 1851-6297 - ISSN 2362-3349 (En LinEA). LAS InICIATIVAS En PRIMERA INFANCIA Bajo análisis. LaURA SANTILLÁN

infancia sea abordada de manera integral. La integralidad, lejos de ser una noción unívoca, opera al menos a través de dos orientaciones. Tal como advierten otros estudios (Barna, 2015), una de las orientaciones que asume la noción de integralidad tiene que ver con enumerar los aspectos de la experiencia infantil que las iniciativas deberían tomar en cuenta a la hora de trabajar en procura de una adecuada atención y desarrollo de los/as niños/as. A tal fin, en diferentes documentos se insta a la necesidad de que el trabajo con los niños más pequeños recupere el aspecto biológico, físico, psíquico, social y emocional de su desarrollo (11). Junto con ello, una segunda acepción del concepto de "integralidad" mucho tiene que ver con la pretensión de desarrollar estrategias de intervención que se alejen de las formas de abordaje clásicas sectoriales de las políticas públicas. En este sentido, la noción de integralidad alude a los tipos de enlaces y cruces intersectoriales que, desde las sugerencias de los organismos internacionales, se espera que se produzca en las políticas públicas. En vinculación con esto último, un rasgo de casi todas las propuestas que se desarrollan dentro y fuera de nuestro país es la articulación entre distintas agencias estatales (educación, salud, cultura, justicia, desarrollo social).

Cuando pasamos de lleno a los contenidos de las propuestas, una serie de cuestiones se ponen de relieve. Una de ellas, tal como anticipamos, es la referencia de esta etapa de la vida, la primera infancia, como un período clave y crucial para sentar las bases del desarrollo futuro de los niños y las niñas. Esta mención, que se presenta de manera prácticamente unánime en todos los documentos, se torna clave para comprender algunas de las coordenadas contemporáneas en cuanto a la atención a los primeros años de la vida. A saber, por un lado, es una referencia que, recuperando alusiones provenientes de la biología, las neurociencias, las ciencias cognitivas y genéticas, sobresale por la eficacia que tiene en instalar una serie delimitada de premisas que se presentan como irrefutables. Entre estas premisas se destaca la referencia reiterada de que los procesos formativos de los seres humanos están fuertemente influidos - cuando no determinados- por las experiencias tempranas en la vida. En la mayoría de las propuestas, estas afirmaciones se sostienen en base a un núcleo cerrado de presupuestos. En las enunciaciones que hegemonizan el campo de intervención en primera infancia -tales como las provenientes de los organismos de cooperación internacionales- uno de los presupuestos es que los dos primeros años de la vida son determinantes en el desarrollo cognitivo y socioemocional de los niños (12):

Los primeros años son decisivos en la vida de todas las personas. Existe un consenso generalizado respecto a la especificidad, complejidad, importancia y velocidad de los procesos madurativos que ocurren en esta etapa de la vida, cuando se constituyen las bases materiales y simbólicas del sujeto [...]. La mayor parte del desarrollo del cerebro ocurre antes de que el 
Revista de la Escuela de Ciencias de la Educación, año 12, nRo. 11, vol. 2, julio a diciembre de 2016. Páginas 217 235. ISSN 1851-6297 - ISSN 2362-3349 (En LínEA). LAS INICIATIVAS EN PRIMERA INFANCIA BAJO ANÁLISIS. LaURA SANTILLÁN

niño cumpla 3 años; entonces, antes de que muchos adultos se percaten de lo que está ocurriendo, las neuronas del niño proliferan, las sinapsis establecen nuevas conexiones con asombrosa velocidad y se marcan pautas para el resto de la vida (Unesco, 2009, p. 21).

Desde la fuerza de estos argumentos -presentados de manera extendida con base a su autoridad científica- se convalida la relevancia de trabajar en la primera infancia (13). Aludimos a una relevancia y necesidad que se legitima no sólo por los beneficios que proveería en el presente, sino fundamentalmente -como vamos advirtiendo- por los que traerían en el futuro.

Llegados hasta aquí, hay entonces tres cuestiones -con sus correspondientes efectos- que nos interesa señalar. Una tiene que ver con esta reiterada alusión a la "ciencia" para legitimar la necesidad de intervenir en los primeros años de la vida. Tal como demostraron también otros estudios (Fonseca, 2011), en temas vinculados a la primera infancia se hacen claramente palpables los usos sociales y políticos del discurso de la ciencia. Entre estos usos se destacan las referencias al determinismo que tienen los primeros años de la vida en la personalidad y el desarrollo adulto. Algo a señalar es que se tratan de referencias que, en simultáneo desde muchas voces se dan por sentadas, otras líneas provenientes del campo científico refutan. Al respecto, es importante tener en cuenta que la inquietud por instalar como norma colectiva la existencia de "un período crítico" en el desarrollo humano, lejos de ser unánime, es descartada desde otros planteos dentro de la ciencia. Nos referimos a planteos que incluso se ubican dentro de las neurociencias (Pérez Alvarez, 2011, Benaráos, et al, 2010) y que denuncian las aspiraciones "cerebro-centristas" de muchos de los enunciados sobre el desarrollo de las personas.

En segundo lugar, afirmaciones como las que se difunden en los planteos que hegemonizan el campo resaltan, tal como adelantamos, por presentar a la atención a la primera infancia como solucionadora de problemas a futuro. Entre otros se alude a desempeños escolares, así también al desarrollo de la personalidad y la sociedad, incluyendo en ello la posibilidad de revertir los ciclos de pobreza:

Asegurar que los niños disfruten durante sus primeros años de las mejores condiciones de vida es una de las mejores inversiones que puede realizar un país que piensa en su futuro y en la posibilidad de ser parte de una economía mundializada que se basa en la fortalezca del capital humano. Garantizar un BUEN COMIENZO ${ }^{14}$ para los niños y niñas argentinos puede contribuir a quebrar los ciclos de la pobreza, la enfermedad y la violencia que afectan al país. (Unicef, 2004).

En relación al modo en que se presentan estas argumentaciones, uno de los rasgos que ponen de manifiesto es el hecho de situar a las acciones en primera infancia como resolutivas "por sí mismas" de las problemáticas 
Revista de la Escuela de Ciencias de la Educación, año 12, nRo. 11, vol. 2, julio a diciembre de 2016. Páginas 217235. ISSN 1851-6297 - ISSN 2362-3349 (EN LINEA). LAS INICIATIVAS EN PRIMERA INFANCIA BAJO ANÁLISIS. LAURA SANTILLÁN

sociales. Como consecuencia, desde planteos como tales, las condiciones materiales que permiten o no el buen desarrollo infantil suelen quedar en un plano secundario, cuando no directamente ausente.

Finalmente, para cerrar el análisis, hay una derivación muy importante a señalar en cuanto a los argumentos desde los cuales se estructuran buena parte de las intervenciones que hegemonizan el campo de intervención en Primera infancia. Cuando se toman en conjunto las fundamentaciones de las iniciativas destinadas a los niños y las niñas, un actor que comienza a ser insoslayable es "la familia". Desde los argumentos que instalan los documentos, si algo parecen querer ordenar estas iniciativas son las maneras en que las familias deben llevar adelante la socialización, la crianza y el cuidado de los/as niños/as.

Con esto último nos introducimos en un tema relevante tal como es el interrogante acerca de quiénes son los sujetos destinatarios de las iniciativas en Primera Infancia. Por cierto, si bien las propuestas -avaladas además en la Convención de los Derechos del Niño- colocan a la niñez como protagonista de las acciones, en el derrotero de las mismas, buena parte de las estrategias de intervención recaen sobre los entornos familiares y las comunidades de origen. Esta avanzada hacia la familia tiene lugar de diversos modos, en simultáneo pone el eje de foco en los grupos sociales pertenecientes a los sectores subalternos.

Por un lado, en las postulaciones sobre primera infancia que hoy cobran mayor difusión, como fuimos adelantando, un común denominador es la tendencia hacia la despotilizacion de las condiciones de vida de las comunidades afectadas en las intervenciones. Con esto no queremos decir que las condiciones objetivas estén totalmente ausentes en los manuales y documentos. Según es posible comprobar, en las fundamentaciones que sistematizan las agencias y organismos intervinientes, la alusión a los recursos económicos constituye un punto de arranque. Organismos internacionales -para traer como ejemplo- desarrollan pormenorizados análisis y diagnósticos sobre las poblaciones. En función de ello, dejan explicitada la importancia de tomar en consideración los recursos materiales con que cuentan las comunidades de origen de los niños para garantizar la atención adecuada a los primeros años de la vida. De ese modo, los problemas ligados con la pobreza (entre otros, relativos a la falta de acceso agua potable, a sistemas adecuados de saneamiento, problemas en las viviendas) quedan de principio explicitados. Sin embargo, nada de esto descarta que en las fundamentaciones y -en concreto- en el detalle de las acciones se vuelva de manera generalizada a definiciones reificadas sobre la familia. La familia, en varios documentos, aparece como agente natural de cuidado, y por ende como facilitadora o no de un adecuado desarrollo de los niños:

La familia es la unidad básica de la sociedad y, como tal, debe reforzarse. 
Revista de la Escuela de Ciencias de la Educación, año 12, nRo. 11, vol. 2, Julio a diciembre de 2016. Páginas 217235. ISSN 1851-6297 - ISSN 2362-3349 (EN LÍNEA). LAS INICIATIVAS EN PRIMERA INFANCIA BAJO ANÁLISIS. LAURA SANTILLÁN

La familia tiene derecho a recibir una protección y un apoyo completos. La responsabilidad primordial de la protección, la educación y el desarrollo de los niños incumbe a la familia. Todas las instituciones de la sociedad deben respetar los derechos de los niños, asegurar su bienestar y prestar la asistencia apropiada a los padres, a las familias, a los tutores legales y a las demás personas encargadas del cuidado de los niños para que éstos puedan crecer y desarrollarse en un entorno seguro y estable y en un ambiente de felicidad, amor y comprensión, teniendo presente que en diferentes sistemas culturales, sociales y políticos existen diversas formas de familia (Unicef, 2005, p. 13).

Con algunas variaciones en las retóricas y las narrativas, uno de los ítems sobre el que vuelven las distintas propuestas es el interés por desarrollar acciones tendientes al fortalecimiento y apuntalamiento de las capacidades de las familias para la crianza de los niños y las niñas. Según aparecen en los documentos analizados, se busca:

Las familias son responsables de la crianza de sus hijos y las naturales proveedoras de cuidado, afecto, estímulo y valores a los niños pequeños de acuerdo a su cultura. Las oportunidades para que los niños logren mayores niveles de desarrollo personal aumentan cuando son favorecidas por políticas públicas y programas de apoyo a la familia formulados con abordajes multidisciplinarios e intersectoriales (Unicef, 2005, p. 14).

Potenciar a las familias y cuidadores primarios para relacionarse con los niños y las niñas de manera más equitativa e inclusiva, e igualmente a los centro de desarrollos infantil y la comunidad, partiendo del respeto por la diversidad cultural en las pautas de crianza (Documento Conpes, Colombia, 2007, p. 28).

Cualquier acción que tenga por objetivo principal contribuir a mejorar las circunstancias en que se desarrollan los niños y las niñas requiere sin lugar a dudas fortalecer a la familia en sus funciones ce crianza, tanto en lo que respecta a la educación, el cuidado y la protección de sus hijos e hijas mediante la generación de vínculos basados en el afecto y el amor. Las influencias más significativas, constantes y duraderas provienen de la familia en la que hemos transitado nuestros primeros años de vida (Programa Primeros Años, Argentina, 2010).

Entonces, junto con la inquietud que exponen tanto los estados nacionales, así como los organismos multilaterales en la ampliación de la cobertura en educación del nivel inicial, un eje central es el apuntalamiento de lo que usualmente se conoce como la socialización primaria, cuyo agente central pareciera no ser otro que el núcleo familiar de los niños. 
Revista de la Escuela de Ciencias de la Educación, año 12, nRo. 11, vol. 2, julio a diciembre de 2016. Páginas 217235. ISSN 1851-6297 - ISSN 2362-3349 (EN LINEA). LAS INICIATIVAS EN PRIMERA INFANCIA BAJO ANÁLISIS. LAURA SANTILLÁN

Desde las propuestas que hegemonizan el campo, se trata de intervenciones sobre las familias que incluyen dispositivos ligados a recuperar y valorizar los conocimientos y saberes locales, como así también las creencias y experiencias con el fin explícito de apuntalar, fortalecer, construir, fomentar y configurar lo que se entiende como "entorno protector y/o seguro". Un punto que no puede pasársenos por alto es que, dentro del amplio colectivo de las familias, las acciones iniciativas -sean éstas de origen gubernamental ó no- tienen como destinatario exclusivo a los hogares pertenecientes a los sectores populares.

Tomadas en conjunto, las directrices sobre la importancia de generar un "entorno seguro", nos lleva de un modo u otro a la apelación a que las familias asuman por sí mismas el compromiso de ser "responsable de los otros" (los/ as niños/as). Siguiendo los aportes que nos proveen otros estudios, aludimos a orientaciones que nos hablan de renovadas racionalidades y formas de regulación de comportamientos sustentados en la autovalía (Rose, et al, 2006). Se trata por cierto de una forma de control y regulación de la familia que -aun en su novedad- se constituye en el marco de una larga historia de intervención sobre las clases subalternas (Santillán, 2012).

Para cerrar, cabe decir que en los escenarios donde investigamos, es posible encontrar un abanico amplio de acciones destinadas al propósito de acompañar y apuntalar la crianza y el cuidado de los niños de más corta edad. Son acciones llevadas adelante por los diversos actores y espacios, y que involucra de manera llena a las familias. Si bien no fue objeto de este estudio dar cuenta de las dinámicas que asumen los postulados generales en los contextos locales, el registro etnográfico que venimos realizando nos conduce a no descartar bajo ningún aspecto las continuas apropiaciones y reconfiguraciones que cotidianamente tiene lugar en escenarios en donde se llevan a cabo las acciones con los niños y las niñas.

\section{Palabras finales}

En este artículo nos propusimos abordar las premisas y construcciones de sentidos que se ponen en juego en la producción documental y legislativa vinculado con el campo de intervención que comienza a definirse bajo la categoría de atención a la "Primera Infancia". Campo que, como advertimos, está conformado por un complejo diverso de actores sociales y políticos: organismos gubernamentales, no gubernamentales e internacionales.

Fue inquietud de este trabajo, no dar por sentado ninguno de los aspectos que hacen a las intervenciones en Primera Infancia. Por ello es que el análisis se propuso sistematizar la documentación de al menos un conjunto de actores que participan, los saberes que construyen y cuáles son algunos efectos de las principales premisas que se formulan.

En cuanto a los actores, nos tomamos de la imagen de polifonía para 
Revista de la Escuela de Ciencias de la Educación, año 12, nRo. 11, vol. 2, Julio a diciembre de 2016. Páginas 217235. ISSN 1851-6297 - ISSN 2362-3349 (EN LínEA). LAS INICIATIVAS EN PRIMERA INFANCIA BAJO ANÁLISIS. LAURA SANTILLÁN

aproximarnos al reconocimiento de la multiplicidad de sujetos colectivos y del tipo de conexiones que tiene lugar en cuanto al campo de intervención en Primera Infancia. Desde nuestro punto de vista, sin dejar de atender a la heterogeneidad de voces y a la naturaleza singular de los distintos protagonistas - portando muchos de ellos banderas contestatarias- un rasgo que advertimos son las importantes continuidades que perviven, al menos en relación a los relatos y las racionalidades que se imponen con fuerte hegemonía. Al respecto, es necesario advertir que en esta multiplicidad de actores, los organismos internacionales (de financiamiento y cooperación) resaltan en la capacidad de fijar acentos y sentidos en el tema. Tal como quedó demostrado en el análisis, en relación a la preeminencia de los organismos internacionales, acordamos con otros estudios acerca de la necesidad de no soslayar las contradicciones y paradojas que encierra "la cooperación" trasnacional (Torres, 2001). Al verticalismo, la tendencia al "recetario" y el endeudamiento que generan, sumamos las advertencias en cuanto a la contribución que las concertaciones y gestiones globales tienen en la pretensión de generar "un pensamiento único" en temas que son álgidos para los distintos países y las distintas poblaciones en cuanto al cuidado y la educación de los/as niños/as.

En ese marco, se hacen inteligibles elementos clave de las formas en que hoy se imprimen algunas racionalidades y saberes en torno a este recorte hiper específico de la "primera infancia". Investidos desde el valor de verdad que se le otorga al discurso científico, las premisas sobre el "período crítico" y la "ventana de oportunidad" (entre otros, en el desarrollo del cerebro), tornan a los postulados que predominan en el campo de intervención en los primeros como años como irrefutables y sin otra alternativa posible.

En continuidad con este punto, uno de los aspectos que nos interesó señalar desde el análisis tiene que ver con los efectos no necesariamente previstos -0 al menos no siempre problematizados- en las intervenciones contemporáneas en Primera Infancia. Aun cuando la referencia más enunciada sean los niños y las niñas, el objeto de buena parte de las sugerencias culmina casi siempre en las familias. Pero no se trata de cualquier familia. Desde un proceso orientado a la regulación moral, el eje central hacia donde se dirige el proyecto de normalización y modelación acerca de los comportamientos y cómo debe llevarse adelante la crianza y el cuidado de los hijos son las familias de los sectores socialmente más desfavorecidos.

Según entendemos, de manera más y menos directa se conciertan una serie de directrices y acciones que, en función de las orientaciones que asumen, nos hablan de formas de control de hábitos familiares bajo formas renovadas de disciplinamiento de los modos de vida. 
Revista de la Escuela de Ciencias de la Educación, año 12, nRo. 11, vol. 2, julio a diciembre de 2016. Páginas 217235. ISSN 1851-6297 - ISSN 2362-3349 (EN LINEA). LAS INICIATIVAS EN PRIMERA INFANCIA BAJO ANÁLISIS. LAURA SANTILLÁN

\section{Notas Bibliográficas}

(1) En el presente escrito la categoría de Primera Infancia no constituye una categoría propia ni analítica, sino que, en tanto categoría social, expresa el modo en que en estos últimos años se ha designado de manera generalizada y hegemónica a los primeros años de la vida de los/as niños/as que no han alcanzado aún la edad de la escolaridad básica (usualmente estipulada en los 6 años).

(2) A nivel internacional, en las legislaciones y programas vigentes, no hay un acuerdo unívoco acerca de las edades que abarca la "Primera Infancia" (esto implica que puede abarbar entre 0 a 4 años, así como de 0 a 8 años). En nuestro país suele primar el período definido desde el nacimiento hasta el ingreso a la educación primaria, es decir de 0 a 5 años.

(3) Esta investigación la llevamos adelante en el marco del Consejo Nacional de Investigaciones Científicas y Técnicas, del cual formamos parte desde el año 2008.

(4) Acepción del Diccionario de uso español María Moliner, ed Grados, 2007.

(5) Programas como éste se han venido proponiendo, a través de la creación de la "Mesa Interministerial de Gestión y Programación del Programa Nacional Primeros Años, el desenvolvimiento de acciones orientadas a favorecer el desarrollo de las niñas y niños de 0 a 4 años.

(6) Aunque excede al análisis de nuestro trabajo es importante decir que todos los continentes quedan comprendidos dentro de este interés por la primera infancia, demostrando las distintas regiones singularidades en los focos de preocupación e intervención. Según dejan testimoniado los documentos analizados, para el caso de Europa, por ejemplo, el foco está puesto en la situación de niños y niñas de las familias migrantes.

(7) Nos referimos a procesos ligados con la democratización, la gobernabilidad, entre otros.

(8) Cabe señalar que las respuestas de los estados nacionales a las concertaciones mundiales no son unívocas. En este mismo encuentro en Dakar, los países de Latinoamérica hicieron pública su insatisfacción en cuanto al progresivo involucramiento de los organismos internacionales que -de ser entidades de financiamiento- comenzaron a ocupar un protagonismo inusitado en el asesoramiento técnico, la investigación, el monitoreo y evaluación de las políticas educativas de los estados. En este pronunciamiento se aludió a la preocupación de un "pensamiento único" instalado en el marco de la "cooperación internacional". A este pronunciamiento luego le siguió la Declaración de Cochabamba en el año 2001 (para profundizar ver: Torres, 2001). En esas contestaciones a las influencias de los organismos internacionales podemos nombrar también a las Cumbres de las Américas, a las reuniones del MERCOSUR y UNASUR, aunque excede las posibilidades del escrito analizar con profundidad los contenidos y alcances concretos logrados al respecto.

(9) Desde su oficialización en 1946 la UNESCO se presenta como uno de los organismos especializados de las Naciones Unidas. Nuestro país, Argentina, es 
Revista de la Escuela de Ciencias de la Educación, año 12, nRo. 11, vol. 2, Julio a diciembre de 2016. Páginas 217235. ISSN 1851-6297 - ISSN 2362-3349 (EN LÍNEA). LAS INICIATIVAS EN PRIMERA INFANCIA BAJO ANÁLISIS. LAURA SANTILLÁN

Estado miembro de la UNESCO desde el año 1948, dos años después de su constitución oficial. Como Estado miembro (o como miembro asociado) los países tienen derecho a percibir las ayuda y colaboraciones que se desarrollen en el seno de la UNESCO, a la vez que el deber de someter a la Organización, en el momento y la forma que decida la Conferencia General, informes sobre las leyes, reglamentos y estadísticas relativos a sus instituciones y actividades educativas, científicas y culturales, así como sobre el curso dado a las recomendaciones y convenciones que se sugirieran.

(10) UNICEF, definido como el Fondo de las Naciones Unidas para la Infancia, desarrolla su labor en más de 190 países y territorios por medio de programas y Comités Nacionales.

(11) Al respecto, en la fundamentación del Programa Chile Crece Contigo, la noción de integral es mencionado en ocasiones como multidimensional.

(12) Ver, entre otros, el Documento Consejo Nacional de Economía social, República de Colombia (2007); fundamentación Chile Contigo Crece.

(13) Para ampliar en los efectos políticos de este uso de la ciencia ver Fonseca, 2011.

(14) El resaltado pertenece al documento.

\section{Referencias bibliográficas}

- Barna, A. (2015). La gestión de la infancia entre lo local y lo global. Una etnografía sobre intervenciones destinadas a "restituir derechos de niños" en dispositivos estatales en las Leyes de Protección Integral. Tesis doctoral sin publicar.

- Benaráos et al. (2010). Neurociencia y educación: hacia la construcción de puentes interactivos. Revista de Neurología, 50 (3), 179-186.

- Cardarelli, G. (2005). Los niños están en todas partes. Modalidades "formales" y "no formales" de atención a la primera infancia. En M. Pereira (Ed.). Intervenciones en primera infancia (pp. 23-44). Buenos Aires: Novedades educativas.

- Corrigan, P y Sayer, S. (2007). El Gran Arco: La formación del Estado inglés como revolución cultura". En Lagos y Calla Antropología del Estado. Dominación y prácticas contestatarias en América Latina (pp.39-116) Bolivia, La Paz: INDH/ PNUD.

- Fairclough, N. (1993). Discourse and Social Change. Cambridge University Press

- Fonseca, C. (2011). Tecnologías globales de la moralidad materna: políticas de la educación para la primera infancia en el Brasil contemporáneo". En Cosse, Llobet, Villalta \& Zapiola: Infancias, políticas y saberes en Argentina y Brasil (Siglo XIX y $X X)$. (pp 175-204). Buenos Aires: El Teseo.

- Foucault, M. (2008). La gubernamentalidad. Em G. Giorgi, y Rodriguez (Comp.). Ensayos sobre biopolítica. Excesos de vida. Buenos Aires: Paidos. p. 187-215.

- Goncalves Nunes, D (2009). Educacao infantil e mundo político. Revista Katal, 12 (1), 86-93. 
Revista de la Escuela de Ciencias de la Educación, año 12, nRo. 11, vol. 2, julio a diciembre de 2016. Páginas 217235. ISSN 1851-6297 - ISSN 2362-3349 (EN LiNEA). LAS INICIATIVAS EN PRIMERA INFANCIA BAJO ANÁLISIS. LAURA SANTILLÁN

- Jaramillo, I (2009). La política de primera infancia y las madres comunitarias. Zona próxima, 11, 86-101.

- Marcus, G. y Fischer, M. (2000). La antropología como crítica cultural. Un momento experimental en las ciencias humanas. Buenos Aires: Amorrortu editores.

- Pérez Alvarez, M. (2011). El magnetismo de las neuroimágenes: moda, mito e ideología del cerebro. Papeles del Psicólogo, 32(2), 98-112-

- Rose et al (2006). Governmentality. Annual Review of Law and Social Science, 6, 83-104.

- Rosemberg, F. (2002). Organizações multilaterais, Estado e políticas de educação infantil. Cadernos de Pesquisa (115), 25-63.

- Santillán, L. (2012): Quiénes educan a los chicos. Infancia, trayectorias educativas y desigualdad. Buenos Aires: Biblos.

- Torres, R. M. (2001). Globalización y educación. "Cooperación internacional en educación en América Latina: ¿parte de la solución o parte del problema?. Cuadernos de Pedagogía (308), pp.69-86.

- Umayahara, M.(2004). En búsqueda de la equidad y calidad de la educación de la primera infancia en América Latina. Revista Latinoamericana de Ciencias Sociales, niñez y juventud. 2 (2), 21-49.

- Voloshinov, V. (2009) [1929]. El marxismo y la filosofía del lenguaje. Buenos Aires: ediciones Godot.

- Young, M y Fujimoto Gomez, G. (2003). Desarrollo Infantil Temprano: lecciones de los programas no formales. Revista Latinoamericana de Ciencias Sociales, Niñez y Juventud, 1 (1), 85-123.

\section{Fuentes}

- BANCO MUNDIAL: Página sobre pobreza y Capital social. www.worldbank.org/ poverty/scapital/index.htm, 2000.

- BANCO MUNDIAL: Documento Programas de desarrollo en la primera infancia (DPI) otorgan soluciones efectivas en función del costo en América Latina, 2009.

- PROGRAMA PRIMEROS AÑOS. Consejo Nacional de Coordinación de Políticas sociales. Presidencia de la Nación. http://www.primerosanios.gov.ar/

- PROGRAMA NACIONAL "CRECIENDO JUNTOS": Ministerio Nacional de educación y Ministerio de Desarrollo Social: http://www.desarrollosocial.gob.ar/ ninez

- UNESCO: Atención y Educación de la Primera Infancia Informe Regional, 2010.

- UNESCO: Primera infancia en América Latina: La situación actual y las respuestas del Estado. Informe sobre tendencias sociales y educativas en América Latina, 2009.

- UNESCO: Marco de acción en Dakar. Educación para todos: cumplir nuestros 
Revista de la Escuela de Ciencias de la Educación, año 12, nRo. 11, vol. 2, julio a diciembre de 2016. Páginas 217235. ISSN 1851-6297 - ISSN 2362-3349 (En LíneA). LAS INICIATIVAS EN PRIMERA Infancia BAJO análisis. LaURA SANTILLÁN

compromisos comunes, 2000.

- UNICEF: Campaña Buen comienzo, julio 2004.

- UNICEF: Crecer juntos para la Primera Infancia. Encuentro regional de políticas integrales 2011, 2012.

- UNICEF: Hacia una política pública en desarrollo infantil temprano. Las buenas prácticas, 2005. 